\title{
INFLUÊNCIA DO NÍVEL DE TENSÃO DA MOLA NA PERCENTAGEM DE ÁGUA DESVIADA E DE ENERGIA ABSORVIDA EM UM ASPERSOR DE IMPACTO
}

\author{
Rubens Duarte Coelho' ${ }^{1}$ Marcus Vinicius Folegatti
}

\begin{abstract}
RESUMO
O objetivo deste trabalho foi determinar a influência do nível de tensão da mola acoplada ao defletor-martelo de um emissor modelo Naan 233/92, no volume de água desviado do jato principal do aspersor e na quantidade de energia absorvida. Ressalta-se a importância desse estudo na elaboração de normas referentes aos ensaios de equipamentos de irrigação (ABNT - Associação Brasileira de Normas Técnicas), assim como, fornecer parâmetros de entrada aos modelos matemáticos de trajetória balística para simulação de desempenho de aspersores em condições diversas de vento e declive na superfície do solo. A ação do defletor-martelo típico em aspersores de impacto, interfere constantemente no jato de água proveniente do bocal do aspersor. Até o presente momento, entretanto, não existe nenhuma orientação normativa quanto ao grau de tensão da mola acoplada ao defletor, o que deixa algumas incertezas no processo de preparo dos aspersores para ensaios laboratoriais e de campo. Os resultados obtidos demonstraram que, em função do período de oscilação do defletor-martelo (regulagem da tensão da mola), do diâmetro do bocal e da pressão de serviço, pode-se ter uma variação entre 4 e $40 \%$ do volume de água desviado e redução de 35 a $55 \%$ da energia disponível ao movimento balístico do volume de água interceptado.
\end{abstract}

Palavras-chave: aspersores, normas de ensaio, irrigação, testes de laboratório

\section{INFLUENCE OF SPRING TENSION ON THE PERCENTAGE OF REDIRECTED WATER AND ABSORBED ENERGY FOR A ROTARY SPRINKLER}

\begin{abstract}
The objective of this study was to quantify the influence of the spring tension, of the hammer of a rotary sprinkler (Naan model 233/92) on the volume of water and hydraulic energy absorbed from the deviated portion of the main jet. The importance of this study stands out in the elaboration of technical standards applied to irrigation equipments (ISO, ASAE, ABNT), seeking to supply inputs to mathematical models of ballistic trajectory, to simulate sprinkler performance under variable conditions of wind and soil surface topography. The action of the oscillating arm (hammer) constantly interferes in the main water jet during the whole time the irrigation system is operated in the field. Until the present moment, no standards exist in relation to the degree of tension of the spring coupled to the hammer, which leaves some uncertainties in the initial process of preparing the sprinklers for laboratory and field tests. The obtained results demonstrated that as a function of the hammer oscillation period (tension of the spring), the nozzle diameter and the operational pressure, a variation can be noticed between 4 and $40 \%$ of the deviated volume of water and a reduction of 35 to $55 \%$ of the available energy to the ballistic movement of the intercepted volume of water.
\end{abstract}

Key words: sprinkler, technical standards, irrigation, laboratory test

\footnotetext{
${ }^{1}$ Professor Doutor, DER/ESALQ/USP. E-mail rdcoelho@carpa.ciagri.usp.br

${ }^{2}$ Professor Livre Docente, DER/ESALQ/USP
} 


\section{INTRODUÇÃO}

Atualmente, a irrigação por aspersão do tipo portátil encontra-se bastante difundida em todo o território nacional, existindo um grande número de aspersores de pequeno e médio portes, irrigando diferentes culturas, nos mais variados níveis tecnológicos. A uniformidade de aplicação de água em um sistema de irrigação do tipo aspersão convencional depende de diversos fatores, dentre os quais se destacam: as regulagens mecânicas disponíveis nos emissores (diâmetro do bocal, tensão da mola do defletor-martelo, ajuste do mancal, dispositivo para ajuste setorial, parafuso quebra-jato etc.), o perfil de distribuição do emissor, o espaçamento entre emissores, a incidência de ventos e a pressão de operação. Pouco se sabe, entretanto, como as regulagens disponíveis nos emissores afetam seu desempenho no campo, uma vez que as condições de ensaio laboratorial nem sempre correspondem à realidade de uso, na prática do dia-a-dia. O caso típico em que se verifica tal afirmação é o ponto de regulagem da mola acoplada ao defletor-martelo. O acúmulo de partículas de solo no mancal dos emissores poderá dificultar, ou mesmo impedir, que o emissor gire; neste caso, é freqüente observar que o irrigante incrementa a pressão na mola, para que a freqüência de oscilação do defletor aumente e, com isto, o aspersor possa girar com maior regularidade.

Na realidade, a maior parte da energia responsável pelo giro do emissor é proveniente do próprio jato d'água. Ao contrário do que se imagina, a tensão da mola não contribui diretamente no processo de rotação do emissor. A força da mola não é suficiente para girar o aspersor, sendo sua função básica promover a entrada do defletor-martelo no jato d'água.

Outro ponto de suma importância no processo de rotação do aspersor é o posicionamento do batente onde o defletor-martelo impulsionado pelo jato d'água irá transferir a energia (torque) para o mancal do aspersor; se o batente não estiver posicionado no ponto exato, no final da primeira posição da peça triangular oscilante, antes de iniciar a segunda posição, o aspersor não irá girar de maneira regular, podendo até, em alguns casos, ficar parado; o ponto exato do posicionamento do batente alterase com o diâmetro do bocal; portanto, aspersores que trabalham com diferentes bocais são os mais propensos a apresentarem problemas de rotação no campo, caso o projeto mecânico não tenha sido exaustivamente analisado para diferentes condições de operação. Observa-se, no aspersor de impacto, que uma parte da energia do jato d'água, interceptada pelo defletor-martelo, é utilizada para aumentar a intensidade de batida do defletor no batente e a outra parte é utilizada na deflexão do conjunto mola + defletor para fora do jato.

Solomon (1979) analisando a variabilidade dos resultados de testes de uniformidade de aspersores, dividiu em duas categorias os fatores que afetam a dispersão dos resultados, em que a primeira envolve imprecisões relacionadas aos volumes de água coletados, à pressão de serviço e à velocidade e direção do vento; a segunda categoria, além de ser a mais importante, explica por que dois aspersores idênticos e nas mesmas condições de ensaio, apresentam resultados de CUC diferentes; isto se deve às pequenas diferenças de ajuste no bocal ou no mecanismo de rotação do emissor, que pode afetar significativamente o que acontece com a água ao deixar o aspersor.
Fischer \& Wallender (1988) estudaram a influência do tamanho do coletor e do tempo de duração dos ensaios, nos valores dos coeficientes de uniformidade empregando, para tanto, um sistema de leitura automatizado dos volumes coletados. Neste estudo, foram comparados os resultados obtidos com coletores apresentando diâmetros internos de 400, 235 e $127 \mathrm{~mm}$ e duração dos testes variando entre 5 e 120 minutos. Nos ensaios conduzidos com um aspersor rotativo do tipo impacto (bocal $3,2 \mathrm{~mm}$ e pressão de $276 \mathrm{kPa}$ ) observaram maior variabilidade de precipitações nos coletores situados próximo ao aspersor e no final do raio de alcance, levantando-se a hipótese de que o impacto do defletor-martelo, desviando água nas proximidades do emissor, aumentou o coeficiente de variação nas referidas regiões. O coeficiente de variação do volume de água coletado diminuiu, a medida em que a duração do teste e o diâmetro dos coletores aumentaram.

Segundo Bernuth \& Gilley (1984) existe pouca informação na literatura quantificando a fração de água desviada e a energia absorvida do jato principal de emissores devido à ação do defletor-martelo. Comentam, também, que engenheiros da indústrias fabricantes supõem que, para aspersores de tamanho médio, $15 \%$ da vazão total são desviados e $50 \%$ da energia de velocidade são absorvidos pelo defletor-martelo. Esses autores citam, ainda, duas fontes na literatura: Kohl (1974) menciona que $51 \%$ da energia são absorvido e Bean (1965) diz que, em condições normais de operação, 13,2\% da vazão são desviados pelo defletor-martelo, sendo que este valor pode variar entre 40 e $50 \%$, caso a freqüência de interrupção do jato seja muito elevada.

O objetivo deste trabalho foi determinar a influência do nível de tensão da mola acoplada ao defletor-martelo do aspersor de impacto modelo Naan 233/92, no volume de água desviado do jato principal do aspersor e na quantidade de energia absorvida, para diferentes pressões de operação do emissor e diâmetros do bocal principal.

\section{MATERIAL E MÉTODOS}

Foram ensaiados três aspersores modelo Naan 233/92, com os seguintes diâmetros de bocais: 3,1 x 2,5, 4,4 x 2,5 e 6,2 x 2,5mm (aspersores 1, 2 e 3, respectivamente). O referido modelo de aspersor possui, na extremidade final do defletor-martelo, uma peça triangular oscilante que contata o jato de água proveniente do bocal principal, tendo como objetivo fazer o aspersor girar regularmente, mesmo em pressões de operação muito baixas. Esta peça oscilante pode assumir duas posições distintas: a primeira posição favorece a entrada do defletor-martelo no jato de água e a segunda induz o defletor-martelo a sair, a transição entre uma e outra posição é efetuada pela ação do jato d'água do aspersor.

Inicialmente, conduziu-se um ensaio de vazão x pressão dos emissores, visando determinar o coeficiente de descarga (Cd) e a relação vazão x pressão dos emissores. Cada aspersor foi submetido a duas baterias de ensaios, a primeira (3 repetições) com os bocais principal e secundário em funcionamento e a segunda (3 repetições) somente com o bocal principal em operação.

Posteriormente, conduziu-se um segundo ensaio para determinação da força tangencial da mola acoplada ao defletormartelo (3 repetições). Observa-se, na Figura 1, a bancada de 
teste utilizada neste ensaio, composta por uma balança digital com $0,01 \%$ de precisão, acoplada a um sistema de torção regulável do mancal do aspersor (defletor-martelo apoiado na balança) de maneira tal, a quantificar a força tangencial da mola para diferentes ângulos de deflexão do defletor-martelo do aspersor.

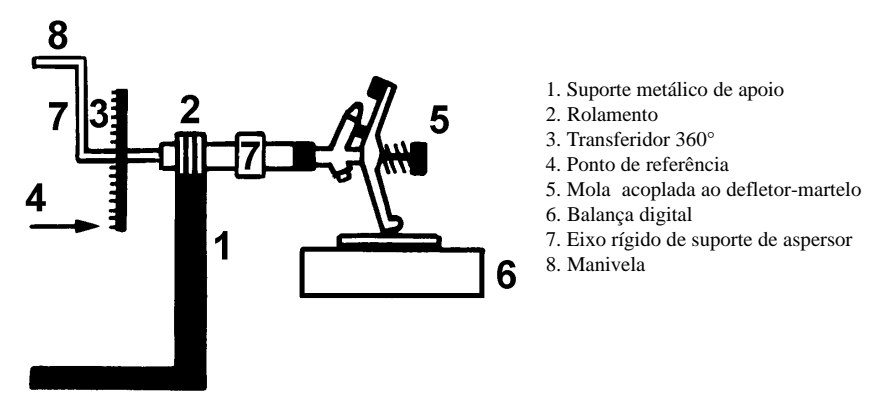

Figura 1. Representação esquemática do ensaio da força tangencial de torção dos aspersores ensaiados

Finalizado o ensaio de torção da mola e com o auxílio de uma cúpula especialmente concebida para os testes de laboratório, deu-se início ao ensaio para determinação do volume de água desviado do jato principal pela ação do defletor-martelo (Figura 2) simultaneamente aos ensaios para determinação do período de oscilação e do ângulo de deflexão máximo do defletor-martelo em operação (Figura 3).

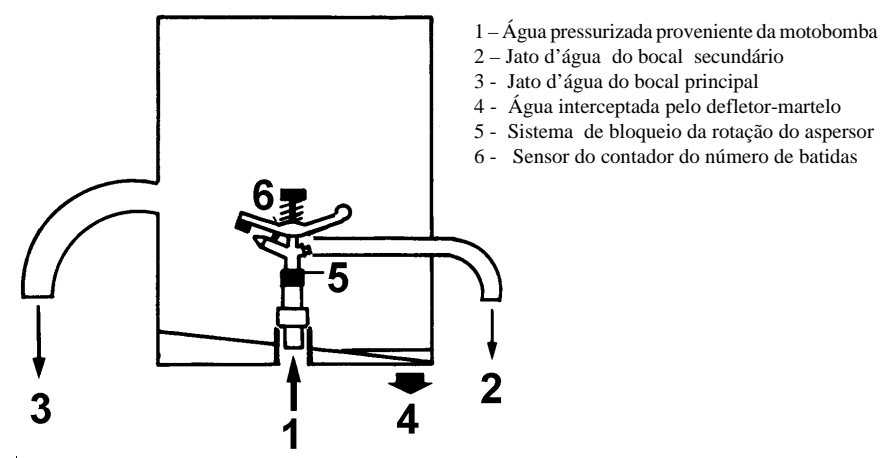

Figura 2. Representação esquemática da cúpula utilizada no ensaio do volume de água desviado e no período de oscilação do defletor-martelo

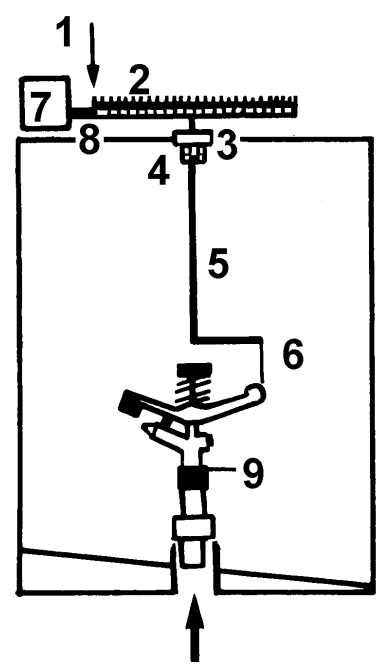

1. Ponto de referência

2. Transferidor sobre polia

3. Rolamento

4. Mini eixo cardã com elemento sensor

5. Extensor do cardã

6. Elemento sensor de deflexão do defletor-martelo

7. Motor elétrico (12 volts)

8. Correia de acoplamento motor-polia

9. Bloqueador de rotação do aspersor

Figura 3. Representação esquemática do ensaio para determinação do ângulo de deflexão do conjunto defletormartelo do aspersor ensaiado
O período de oscilação foi quantificado com auxílio de um contador eletrônico de batidas, acoplado ao batente do aspersor de acordo com o esquema da Figura 2; observa-se também, nesta figura, que o movimento de rotação do aspersor foi bloqueado, para que as vazões dos bocais principal e secundário pudessem ser canalizadas para fora da cúpula, sendo que apenas a água interceptada pelo defletor-martelo ficasse retida no interior da cúpula e dirigida para um ponto de coleta.

O grau máximo de deflexão do defletor-martelo foi mensurado com auxílio de um sensor de deflexão (Figura 3) acoplado a um transferidor de $360^{\circ}$, localizado na parte superior da cúpula idealizada. Por convenção, adotou-se, como Tensão 1, a menor tensão da mola, que possibilitou ao aspersor trabalhar regularmente (velocidade de giro uniforme); as demais tensões foram estabelecidas por deflexões acumuladas de $60^{\circ}$ (intervalo mínimo de regulagem) resultando os seguintes níveis: Tensão 0 - defletor fora da ação do jato, Tensão 1 - 60 , Tensão 2 - $120^{\circ}$, Tensão $3-180^{\circ}$ etc.

Pelo ângulo de deflexão da mola, foi possível calcular-se a energia gasta para a realização deste movimento porém, conforme já mencionado, esta é apenas uma parte da energia capturada; a mensuração da outra parte exigiria equipamentos mais sofisticados, não disponíveis no ensaio realizado; sendo assim e face ao elevado grau de simetria da peça triangular oscilante assumiu-se, nos cálculos efetuados, que a energia total absorvida pelo defletor-martelo equivale a duas vezes a energia gasta na deflexão da mola; não se levou em consideração o atrito no eixo do defletor-martelo nem o coeficiente de elasticidade do choque com o batente (choque inelástico/batente amortecedor).

Calculando-se o trabalho realizado, por meio do produto entre a força e o deslocamento induzido em um movimento circular (Equação 1) pode-se estimar a energia parcial absorvida pelo defletor-martelo a partir do volume de água desviado.

em que:

$$
\zeta=\frac{\mathrm{F} \cdot 2 \cdot \pi \cdot \mathrm{r} \cdot \alpha}{360}
$$

$\zeta$ - trabalho

$\mathrm{F}$ - força tangencial da mola

$r$ - raio do defletor

$\alpha$ - ângulo máximo defletido

O ensaio do coeficiente de elasticidade da mola de torção do aspersor forneceu a força tangencial exercida pelo conjunto mola + defletor-martelo, na distância em que o jato d'água contata o defletor-martelo (ponto de transferência de energia do jato para a peça triangular oscilante); a mensuração da força tangencial na mola nesta distância é muito importante pois o torque é proporcional ao raio (estimativa da energia absorvida).

Entre a posição inicial da mola (defletor encostado no batente) e a posição final (máxima deflexão do defletor) ocorre uma variação linear entre a força tangencial e o grau de deslocamento do defletor. Por se tratar de uma variação linear, a integral do trabalho (energia parcial capturada - EPC) resume-se ao produto entre a força média da mola nos dois pontos considerados e o arco descrito pelo defletor (deslocamento), Equação 2. A energia total capturada (ETC) como descrito anteriormente, foi calculada em função da energia parcialmente capturada (Equação 3).

$$
\mathrm{EPC}=\left(\frac{\mathrm{FTI}+\mathrm{FTF}}{2}\right) . \mathrm{AD}
$$

$\mathrm{ETC}=2 . \mathrm{EPC}$ 
em que :

EPC - energia parcial capturada

FTI - força tangencial inicial

FTF - força tangencial final

AD - arco descrito pelo defletor

ETC - energia total capturada pelo defletor-martelo a partir do volume de água desviado do jato principal.

Pela relação entre a energia total capturada (ETC) e a energia hidráulica disponível no bocal do aspersor (EHD) obtida pelo teorema de Bernoulli (Equação 4) resultou a porcentagem de energia capturada pelo defletor-martelo do aspersor (PECD Equação 5).

$$
\begin{gathered}
\frac{\mathrm{v}_{1}^{2}}{2 \cdot \mathrm{g}}+\frac{\mathrm{P}_{1}}{\gamma_{1}}+\mathrm{z}_{1}=\frac{\mathrm{v}_{2}^{2}}{2 \cdot \mathrm{g}}+\frac{\mathrm{P}_{2}}{\gamma_{2}}+\mathrm{z}_{2} \\
\mathrm{PECD}=\frac{\mathrm{ETC}}{\mathrm{ED}} \cdot 100
\end{gathered}
$$

\section{RESULTADOS E DISCUSSÃO}

Observa-se, na Figura 4, a porcentagem de água desviada do jato principal dos aspersores 1, 2 e 3 devido à ação do defletor-martelo; os resultados apresentam-se distribuídos em seis classes de pressão $(200,250,300,350,400$ e $450 \mathrm{kPa})$ de acordo com o bocal e a tensão da mola utilizada. Cada ponto do gráfico é um valor médio de 3 repetições de cada aspersor: 1, 2 e 3. Nos resultados obtidos, observaram-se os seguintes aspectos:

a) a ação do defletor-martelo no desvio de água do jato principal é mais pronunciada em bocais de menor diâmetro. $\mathrm{Na}$ tensão 4 da mola e na pressão de serviço de $200 \mathrm{kPa}$, observaram-se desvios da vazão do jato principal de 8,3,14,0 e $21,0 \%$ para os bocais principais de $6,2,4,4$ e $3,1 \mathrm{~mm}$ de diâmetros (aspersores 1, 2 e 3, respectivamente);

b) a tensão da mola altera significativamente a porcentagem de água desviada do jato principal. Observando-se o comportamento do bocal 3,1 mm de diâmetro na pressão de $200 \mathrm{kPa}$, nota-se que na tensão 1 da mola foram desviados $10 \%$ da vazão total, enquanto na tensão 8 ocorreu um desvio total de 40\%;

c) quanto menor a pressão de serviço, maior a porcentagem de volume de água desviado do bocal principal; para o bocal de $3,1 \mathrm{~mm}$ de diâmetro na tensão 4 da mola e na pressão de $200 \mathrm{kPa}$, observou-se desvio de $20 \%$, e na pressão de $45 \mathrm{kPa}$, desvio de $10 \%$, explicado pelo fato de que nas menores pressões ocorre menor deslocamento angular do defletor-martelo, reduzindo o período de oscilação e aumentando, conseqüentemente, a porcentagem de volume de água desviado;

d) em condições normais de operação do aspersor (tensão 1 da mola) a porcentagem do volume desviado variou entre 4 e 10\%; já em condiçõos de elevada tensão da mola (tensão 8) este valor oscilou entre 8 e $40 \%$.

Observa-se, na Figura 5, a porcentagem de energia absorvida da água desviada pelo defletor-martelo, em relação à tensão da mola, nas três pressões de serviço, para os três bocais estudados; nesta figura, nota-se nítida variação de comportamento do bocal de $3,1 \mathrm{~mm}$ comparado aos bocais de 4,4 e 6,2mm, levantando-se a hipótese de interação diferenciada entre o jato emergente de cada bocal e a peça triangular oscilante, acoplada ao defletor-martelo dos aspersores ensaiados.

O bocal de $3,1 \mathrm{~mm}$ de diâmetro, inversamente aos outros dois bocais $(4,4$ e $6,2 \mathrm{~mm})$ apresentou menor porcentagem de

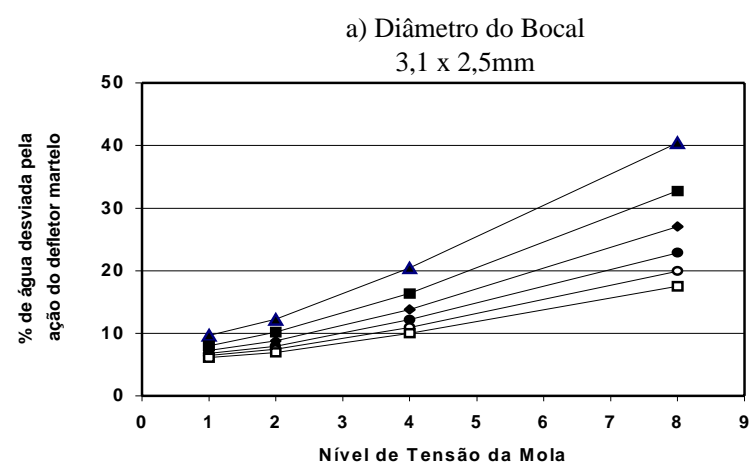

b) Diâmetro do Bocal

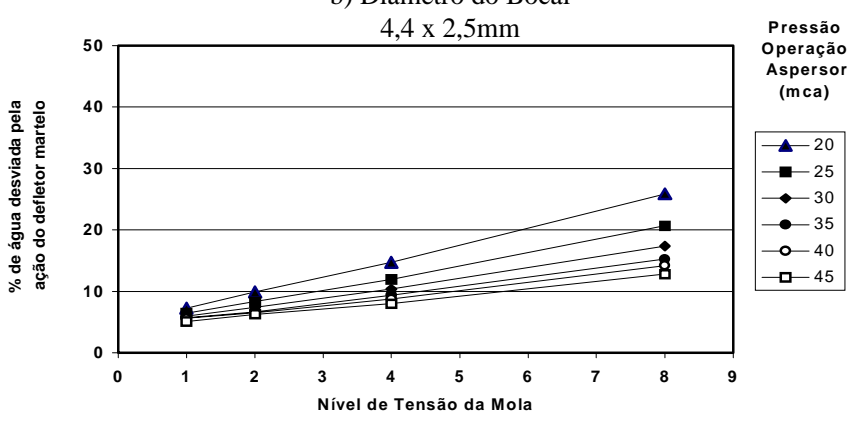

c) Diâmetro do Bocal

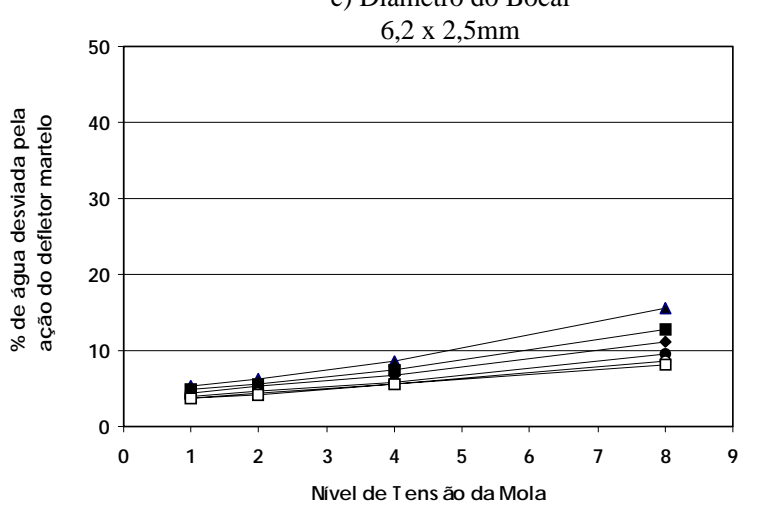

Figura 4. Porcentagem média de água desviada do jato principal dos aspersores ensaiados em relação à tensão da mola acoplada ao defletor-martelo, para diferentes pressões de operação dos aspersores e diâmetros do bocal principal

energia absorvida com o aumento da tensão da mola (exceto na transição da tensão 2 para a tensão 3). A hipótese da interação diferenciada baseia-se no fato de haver inúmeros fatores influenciando o processo, dentre os quais se destacam a inércia do defletor-martelo, a elasticidade do choque com o batente e o posicionamento do eixo de oscilação da peça triangular em relação ao empuxo do jato.

Embora tenha ocorrido variação de comportamento entre os bocais analisados, observou-se uma pequena amplitude de variação da energia absorvida $(30-60 \%)$. Em modelos de simulação de trajetória balística, o emprego de um valor médio de $45 \%$ seria o mais recomendado (energia absorvida do volume desviado).

Com relação ao volume de água desviado pelo defletormartelo observou se, no decorrer dos ensaios realizados, que os aspersores, trabalhando em perfeita sintonia mecânica (giro uniforme, batente bem posicionado, mancal livre etc.) apresentam o defletor-martelo desviando entre 5 a $15 \%$ da vazão total do bocal principal; quando esta percentagem é maior, algum problema mecânico está ocorrendo no emissor. Recomenda-se que seja incluído, nas normas nacionais de 


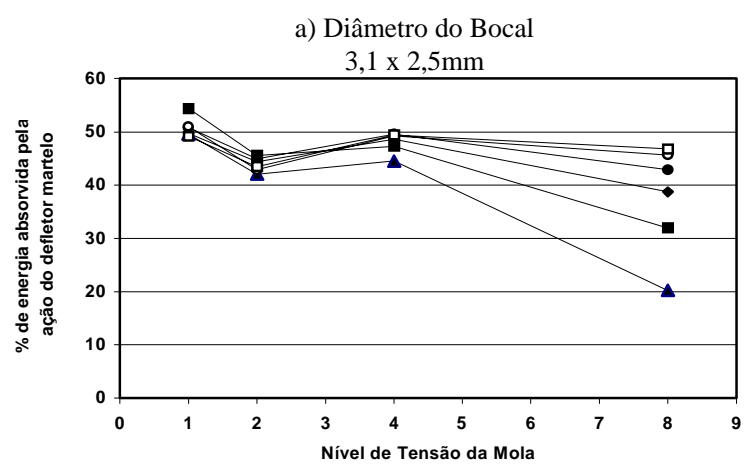

b) Diâmetro do Bocal

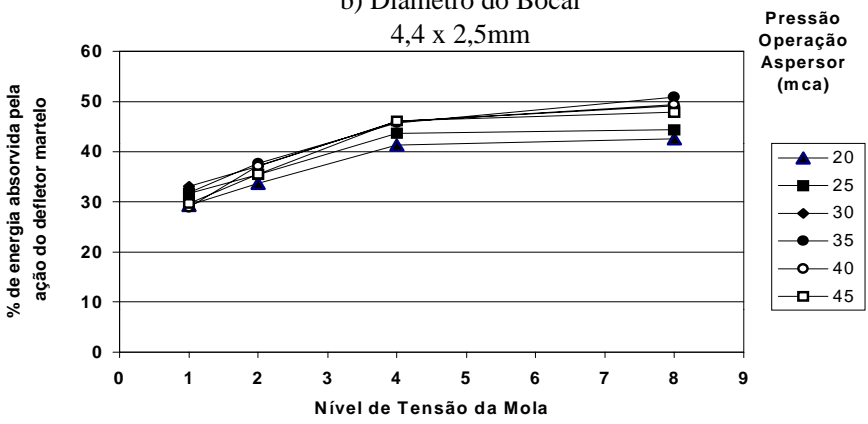

b) Diâmetro do Bocal $6,2 \times 2,5 \mathrm{~mm}$

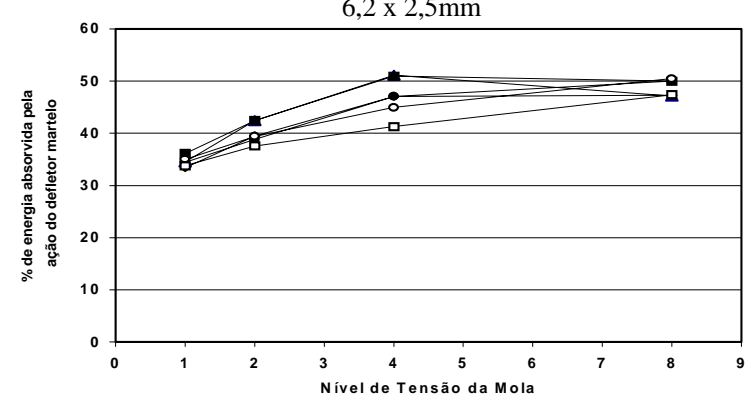

Figura 5. Porcentagem média de energia absorvida da água desviada pelo defletor martelo, em função da tensão da mola acoplada ao defletor-martelo, para diferentes pressões de operação dos aspersores e diâmetros do bocal principal

ensaios de aspersores (ABNT) para fins de certificação, um valor máximo admissível, de volume desviado pelo defletor martelo de $15 \%$ da vazão do bocal principal do aspersor.

Os resultados obtidos nos ensaios aproximaram-se dos valores apresentados na escassa literatura disponível sobre o assunto, conforme se observa na Tabela 1, o que comprova a adequação da metodologia proposta neste trabalho.

Tabela 1. Comparação entre resultados obtidos nos ensaios e citados em literatura

\begin{tabular}{|c|c|c|c|c|}
\hline \multirow{3}{*}{$\begin{array}{c}\text { Valores } \\
\text { Comparativos }\end{array}$} & \multicolumn{2}{|c|}{$\begin{array}{l}\text { \% Vazão desviada pela } \\
\text { ação do defletor-martelo }\end{array}$} & \multicolumn{2}{|c|}{$\begin{array}{c}\text { \% Energia absorvida do } \\
\text { volume d'água desviado } \\
\text { pelo defletor-martelo }\end{array}$} \\
\hline & \multirow{2}{*}{$\begin{array}{c}\text { Cond. } \\
\text { Normais }\end{array}$} & \multirow{2}{*}{$\begin{array}{l}\text { Cond. } \\
\text { Extremas }\end{array}$} & Cond. & Cond. \\
\hline & & & Normais & Extremas \\
\hline Resultados & & & & \\
\hline obtidos & $5-10 \%$ & $4-40 \%$ & $45 \%$ & $35-55 \%$ \\
\hline \multicolumn{5}{|l|}{ ensaios realizados } \\
\hline \multicolumn{5}{|l|}{ Valores citados } \\
\hline na literatura & $13 \%$ & $40-50 \%$ & $50 \%$ & - \\
\hline apresentada & & & & \\
\hline
\end{tabular}

\section{CONCLUSÕES}

De acordo com os resultados obtidos nos ensaios laboratoriais do aspersor modelo Naan 233/92 (diâmetro de bocais: $3,1 \times 2,5,4,4$ x 2,5 e 6,2 x 2,5mm), chegaram-se às seguintes conclusões:

1. O nível de tensão da mola acoplada ao defletor-martelo (braço oscilante) de um aspersor de impacto, altera significativamente o volume de água desviado do bocal principal do emissor, podendo atingir valores entre 4 e $40 \%$ da vazão total, dependendo do diâmetro do bocal e da pressão de operação.

2. A ação do defletor-martelo no desvio de água do jato principal é mais pronunciada em bocais de menor diâmetro. O menor bocal ensaiado $(3,1 \mathrm{~mm}$ de diâmetro) apresentou a maior variação de volume desviado: $10 \%$ de desvio de vazão na tensão 1 da mola e $40 \%$ de desvio na tensão 8 .

3. Quanto menor a pressão de serviço, maior a porcentagem de volume de água desviado do bocal principal explicado pelo fato de que, nas menores pressões, ocorre menor deslocamento angular do defletor-martelo, reduzindo o período de oscilação e aumentando, conseqüentemente, a porcentagem de volume de água desviado.

4. A ação do defletor-martelo absorve entre 35 e $55 \%$ da energia hidráulica do volume de água desviado do jato principal.

5. Observou-se, no decorrer dos ensaios realizados, que os aspersores, trabalhando em perfeita sintonia mecânica (giro uniforme, batente bem posicionado, mancal livre etc.) apresentam o defletor-martelo desviando entre 5 a $15 \%$ da vazão total do bocal principal; quando esta percentagem é maior, algum problema mecânico está ocorrendo no emissor. Recomenda-se que seja incluído, nas normas nacionais de ensaios de aspersores (ABNT) para fins de certificação, um valor máximo admissível de volume desviado pelo defletor martelo, de $15 \%$ da vazão do bocal principal do aspersor.

\section{REFERÊNCIAS BIBLIOGRÁFICAS}

BEAN, A.G.M. Water distribution by irrigation sprinklers. Journal of Agricultural Enginneering Research, Cambridge - UK, v.10, p.314-321, 1965.

BERNUTH, R.D.; Gilley, J.R. Sprinkler droplet size distribution estimation from single leg test data. Transactions of the ASAE, St. Joseph, v.27, n.5, p.1435-1441, 1984.

FISCHER, G.R.; Wallender, W.W. Collector size and test duration effects on sprinkler water distribution measurement. Transactions of the ASAE, St. Joseph, v.31, n.2, p.538- 542, 1988.

KOHL, R.A. Drop size distribution from medium-sized agricultural sprinklers. Transactions of the ASAE, St. Joseph, v.15, n.2, p.690-693, 1974.

SOLOMON, K. Variability of sprinkler coefficient of uniformity test results. Transactions of the ASAE, St. Joseph, v. 22, n.5, p.1078-1080, 1979. 